\title{
Biogenese der mitochondrialen Proteinsynthesemaschine
}

ELISA HANITSCH, RICARDA RICHTER-DENNERLEIN

INSTITUT FÜR ZELLBIOCHEMIE, UNIVERSITÄTSMEDIZIN GÖTTINGEN

The biogenesis and function of the mitoribosome is of central importance as mutations in the respective genes of translation factors, mitoribosomal proteins and ribosome assembly factors lead to impaired mitochondrial translation, oxidative phosphorylation deficiency and subsequently to severe human mitochondrial diseases. Thus, it is of high interest to understand the biogenesis of the mitoribosome and to define the assembly factors and their functions within this complex process.

DOI: $10.1007 / \mathrm{s} 12268-020-1329-\mathrm{y}$

(C) Die Autorinnen 2020

Mitochondrien produzieren den größten Teil der zellulären Energie in Form von ATP

durch oxidative Phosphorylierung (OXPHOS). Dysfunktionen von Mitochondrien führen oft

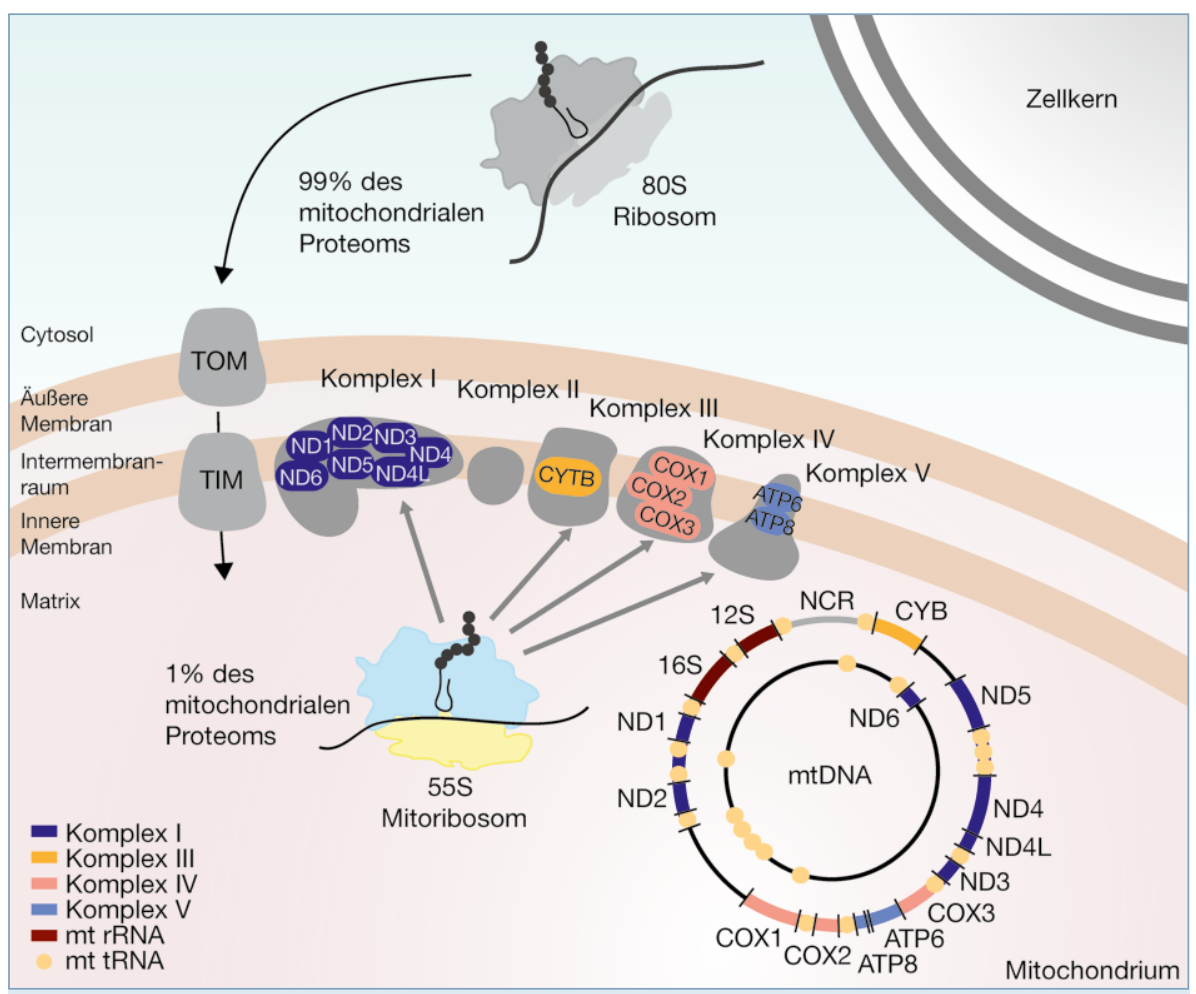

$\Delta$ Abb. 1: Mitochondriale Genexpression. Das mitochondriale Genom (mtDNA) codiert für zwei rRNAs, 22 tRNAs und 13 Proteine, die vom 55S-Mitoribosom synthetisiert werden. Weitere mitochondriale Proteine werden durch cytosolische 80S-Ribosomen synthetisiert und anschließend über die TOM-TIM(translocase of the outer/inner mitochondrial membrane)-Importmaschinerien in die Mitochondrien transportiert. zu schwerwiegenden humanen Erkrankungen, die vor allem Gewebe und Organe mit hohem Energiebedarf betreffen. Diese lebenswichtigen Organellen bakteriellen Ursprungs beinhalten ein reduziertes Genom (mtDNA), das für zwei ribosomale RNAs, 22 tRNAs und 13 essenzielle OXPHOS-Untereinheiten codiert (Abb. 1). Der Großteil der mitochondrialen Proteine ist jedoch im Kern codiert, wird im Cytosol synthetisiert und anschlieBend in die Mitochondrien importiert. Ebendies trifft auch auf alle beteiligten Faktoren $z u$, die für die Expression der mtDNA notwendig sind. Sie umfassen ungefähr 25 Prozent des gesamten mitochondrialen Proteoms, einschließlich aller mitoribosomaler Proteine [1]. Das mitochondriale Ribosom (Mitoribosom) ist von zentraler Bedeutung, da Fehlfunktionen zu mitochondrialen Translationsdefekten, zu einer OXPHOS-Defizienz und folglich zu einer Dysfunktion der Mitochondrien mit klinischer Manifestation führen können. Seit einiger Zeit werden vermehrt Patienten identifiziert, die Mutationen in Genen mitoribosomaler Proteine aufweisen. Aus diesem Grund rückte das Mitoribosom in den letzten Jahren zunehmend in den Fokus der Wissenschaft, was sich auch in einem Anstieg der Veröffentlichungen hochauflösender Strukturanalysen zeigt [2]. Dabei wurden die Unterschiede zwischen dem bakteriellen und dem mitochondrialen Ribosom offensichtlich. Das humane 55S-Mitoribosom setzt sich aus einer kleinen ribosomalen 28S-Untereinheit (mtSSU) und einer großen ribosomalen 39S-Untereinheit (mtLSU) zusammen. Im Laufe der Evolution hat sich der RNA-Gehalt des Mitoribosoms reduziert, einhergehend mit einem Anstieg des Proteinanteils, wodurch sich das RNA/ Protein-Verhältnis im Vergleich zum bakteriellen Ribosom umgekehrt hat. Somit ist es nicht verwunderlich, dass 50 Prozent der mitoribosomalen Proteine kein Homolog im 70S-Ribosom finden. Das humane Mitoribosom weist durch diese strukturellen Veränderungen im Gegensatz zum bakteriellen Ribosom eine höhere Porosität und ein 
um 0,4 Megadalton höheres Molekulargewicht auf.

Diese signifikanten Unterschiede in Struktur und Komposition lassen auch Unterschiede in der Assemblierung vermuten, wobei ein Bedarf an zusätzlichen Assemblierungsfaktoren besteht. Für die Biogenese von Ribosomen in vivo sind Kofaktoren wie RNAHelikasen, RNA-modifizierende Enzyme, Chaperone und GTPasen notwendig.

\section{Biogenese der 28S-Ribo-} somenuntereinheit

Die humane 28S-mtSSU setzt sich aus der mtDNA-codierten ribosomalen 12S-rRNA und den 30 Kern-codierten ribosomalen Proteinen zusammen. Für die Funktion bzw. Stabilität des Ribosoms sind Modifikationen der 12S-rRNA notwendig. Dazu gehören die Methylierung von Cytosinen an den Positionen 839 sowie $841 \quad\left(\mathrm{~m}^{4} \mathrm{C} 839\right.$, $\left.\mathrm{m}^{5} \mathrm{C} 841\right)$, welche durch die Methyltransferasen METTL15 bzw. NSUN4 katalysiert werden, außerdem die Methylierung von zwei Adeninen an den Positionen 936 und 937 der 3'-Schleife der 12S-rRNA $\left(\mathrm{m}_{2}^{6} \mathrm{~A} 936, \mathrm{~m}^{6}{ }_{2} \mathrm{~A} 937\right)$ durch die Methyltransferase TFB1M und die Methylierung von Uridin an der Position 429 ( $\left.{ }^{5} \mathrm{U} 429\right)$ durch TRMT2B (Abb. 2, [3-5]). Fehlfunktionen dieser Methyltransferasen führen zu Assemblierungsdefekten und folglich zum Verlust der mitochondrialen Translation und $\mathrm{zu}$ einer OXPHOS-Defizienz. Um die 12S-rRNA während des Assemblierungsprozesses zu schützen und Methylierungen zu begünstigen, bedarf es dem RNA-Chaperon ERAL1 sowie mtRBFA, die mit der Helix 45 interagieren $[6,7]$. Des Weiteren interagiert die GTPase C4ORF14/ NOA1 mit der 28S-mtSSU, deren Verlust zu Assemblierungsdefekten und somit $\mathrm{zu}$ einem drastischen Verlust der mitochondrialen Translation führt [3]. Die Bedeutung dieser Assemblierungsfaktoren wird auch durch zahlreiche in vivo-
Studien deutlich, die zeigen, dass TFB1M, NSUN4 oder C4ORF14 essenziell während der embryonalen Entwicklung sind.

\section{Biogenese der 39S-Ribosomenuntereinheit}

Die 39S-mtLSU besteht aus der mtDNAcodierten 16S-rRNA und 52 Kern-codierten ribosomalen Proteinen. Bemerkenswert ist, dass die 5S-rRNA, wie sie im bakteriellen Ribosom vorkommt, durch eine tRNA
(tRNA ${ }^{\text {Val }}$ oder tRNA ${ }^{\text {Phe }}$ ) in der mtLSU ersetzt wurde [2]. Ebenso wie die 12S-rRNA erhält auch die 16S-rRNA Modifikationen (Abb. 2, [3]). Die Methyltransferase TRMT61B methyliert ein Adenin an Position 947 ( ${ }^{1}$ A947) in der Helix 71. RPUSD4 katalysiert die Isomerisierung von Uridin zu Pseudouridin an Position 1397 der 16S-rRNA (Psi1397) und an Position 39 der tRNA ${ }^{\text {Phe }}$. Des Weiteren weist die 16S-rRNA drei 2'-O-Ribose-Methylierungen auf (Gm1145, Um1369 und 


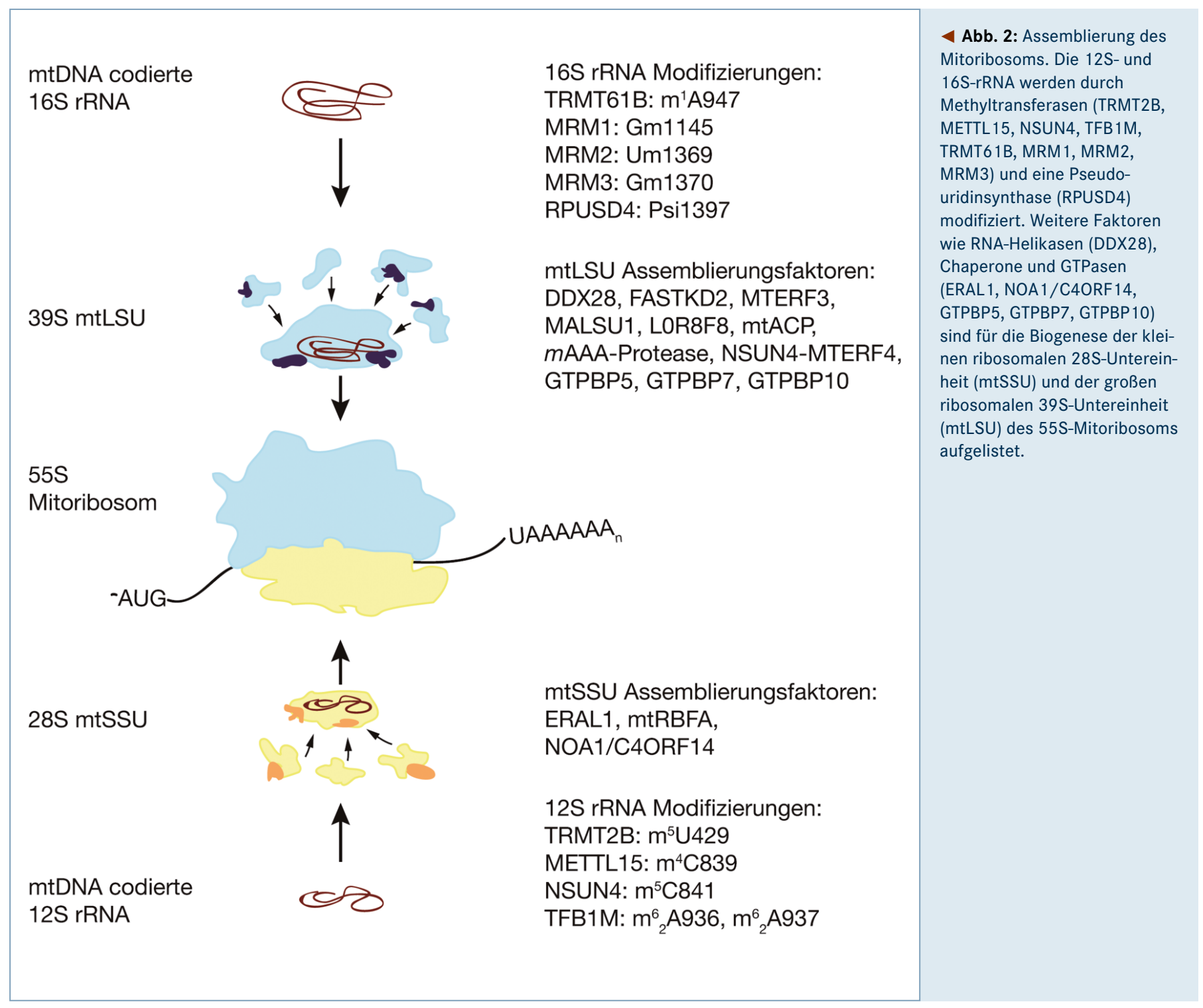

Gm1370), die durch MRM1, MRM2 und MRM3 katalysiert werden. Zusätzlich wurden Faktoren identifiziert, welche für die Stabilität der 16S-rRNA essenziell sind. Dazu zählen die RNA-Helikase DDX28, FASTKD2 sowie MTERF3 [3]. Der Verlust dieser Faktoren führt zu Defekten in der Assemblierung der mtLSU, wobei die molekulare Funktion dieser Proteine noch unklar ist. Auch die mAAA-Protease als Bestandteil der mitochondrialen Qualitätskontrolle ist aufgrund der Prozessierung des ribosomalen Proteins bL32m für die Assemblierung der mtLSU und somit für die mitochondriale Translation erforderlich [8]. Des Weiteren wurden MALSU1, L0R8F8 sowie mtACP in einem späten Assemblierungsintermediat der mtLSU mittels Kryo-Elektronenmikroskopie identifiziert [9]. Auch die Funktion dieser Faktoren ist noch unbekannt, allerdings führt die Depletion von MALSU1 zu Assemblierungs- defekten der mtLSU [3]. Das Protein NSUN4 hat in der Assemblierung eine duale Funktion inne: Es wird nicht nur für die Methylierung der 12S-rRNA benötigt, sondern spielt auch zusammen mit MTERF4 in einem späten Reifestadium der mtLSU eine Rolle und ist für die Formierung des 55S-Mitoribosoms essenziell [3].

\section{Die Rolle der GTPasen in der mtLSU-Assemblierung}

Eine Gruppe von Proteinen, die zunehmend in ihrer Funktion als Regulatoren der Ribosomenbiogenese an Aufmerksamkeit gewinnt, sind die GTPasen. GTPBP5 (OBGH1) und GTPBP7 (MTG1) interagieren mit der mtLSU, wobei die Funktion von GTPBP5, das homolog zum bakteriellen ObgE ist, bisher noch nicht näher beschrieben ist. GTPBP7 ist in den Prozess der Qualitätskontrolle involviert, da es durch seine Interaktion mit
uL19m (Protein der mtLSU) und mS27 (Protein der mtSSU) die Formierung des Monosoms begünstigt [10]. GTPBP10 ist ein weiteres Mitglied dieser Familie und wie GTPBP5 ein ObgE-Homolog. Wir haben gezeigt, dass der Verlust von GTPBP10 zu einer signifikanten Reduktion der mitochondrialen Translation und zu einem verminderten Zellwachstum führt [11]. GTPBP10 interagiert ausschließlich mit der mtLSU, jedoch nicht mit der mtSSU oder dem 55S-Monosom. Durch die Definition des GTPBP10-Interaktoms haben wir weitere Assemblierungsfaktoren als GTPBP10-Interaktoren identifiziert. Hierzu zählen MALSU1, LOR8F8, MTERF4 und NSUN4, die eine Rolle in der späten mtLSUAssemblierung spielen. Der Verlust von GTPBP10 führt zu einer drastischen Reduktion von translatierenden 55S-Monosomen und zur Akkumulation der mtLSU und mtSSU. Jedoch ist eine Anreicherung von frühen 
mtLSU-Assemblierungsintermediaten nicht ersichtlich. Unsere Daten und die anderer Forschungsgruppen haben gezeigt, dass GTPBP10 für späte Reifungsprozesse der mtLSU notwendig ist [11, 12]. Um die detaillierte Funktion von GTPBP10 zu definieren, werden strukturelle Analysen des GTPBP10-mtLSU-Komplexes notwendig sein.

In den letzten Jahren wurden zahlreiche Strukturanalysen der 28S-mtSSU, der 39S-mtLSU und des 55S-Mitoribosoms veröffentlicht, die Aufschlüsse über die Position und Interaktionen einzelner mitoribosomaler Proteine ermöglichen. Allerdings ist wenig über die Biogenese des Mitoribosoms bekannt sowie über die Funktion der erforderlichen Assemblierungsfaktoren. Aus diesem Grund ist es notwendig, bekannte Faktoren zu charakterisieren, weitere zu identifizieren sowie ihre zeitliche Reihenfolge im Assemblierungsprozess zu bestimmen.

\section{Literatur}

[1] Morgenstern M, Stiller SB, Lübbert P et al. (2017) Definition of a high-confidence mitochondrial proteome at quantitative scale. Cell Rep 19:2836-2852

[2] Greber BJ, Ban N (2016) Structure and function of the mitochondrial ribosome. Annu Rev Biochem 85:103-132 [3] Pearce SF, Rebelo-Guiomar P, D'Souza AR et al. (2017) Regulation of mammalian mitochondrial gene expression: recent advances. Trends Biochem Sci 42:625-639

[4] Van Haute L, Hendrick AG, D'Souza AR et al. (2019) METTL15 introduces N4-methylcytidine into human mitochondrial 12S rRNA and is required for mitoribosome biogenesis. Nucleic Acids Res 47:10267-10281 [5] Laptev I, Shvetsova E, Levitskii S et al. (2019) Mouse Trmt2B protein is a dual specific mitochondrial metyltransferase responsible for m5U formation in both tRNA and rRNA. RNA Biol, doi:

10.1080/15476286.2019.1694733

[6] Dennerlein S, Rozanska A, Wydro M et al. (2010) Human ERAL1 is a mitochondrial RNA chaperone involved in the assembly of the $28 \mathrm{~S}$ small mitochondrial ribosomal subunit. Biochem J 430:551-558
[7] Rozanska A, Richter-Dennerlein R, Rorbach J et al. (2017) The human RNA-binding protein RBFA promotes the maturation of the mitochondrial ribosome. Biochem J 474:2145-2158

[8] Nolden M, Ehses S, Koppen M et al. (2005) The m-AAA protease defective in hereditary spastic paraplegia controls ribosome assembly in mitochondria. Cell 123:277-289

[9] Brown A, Rathore S, Kimanius D et al. (2017) Structures of the human mitochondrial ribosome in native states of assembly. Nat Struct Mol Biol 24:866869

[10] Kim H-J, Barrientos A (2018) MTG1 couples mitoribosome large subunit assembly with intersubunit bridge formation. Nucleic Acids Res 46:8435-8453

[11] Lavdovskaia E, Kolander E, Steube E et al. (2018) The human Obg protein GTPBP10 is involved in mitoribosomal biogenesis. Nucleic Acids Res 46:8471-8482 [12] Maiti P, Kim H-J, Tu Y-T et al. (2018) Human GTPBP10 is required for mitoribosome maturation. Nucleic Acids Res 46:11423-11437

Funding: Open Access funding provided by Projekt DEAL. Open Access: Dieser Artikel wird unter der Creative Commons Namensnennung 4.0 International Lizenz veröffentlicht, welche die Nutzung, Vervielfältigung, Bearbeitung, Verbreitung und Wiedergabe in
jeglichem Medium und Format erlaubt, sofern Sie den/die ursprünglichen Autor(en) und die Quelle ordnungsgemäß nennen, einen Link zur Creative Commons Lizenz beifügen und angeben, ob Änderungen vorgenommen wurden. Die in diesem Artikel enthaltenen Bilder und sonstiges Drittmaterial unterliegen ebenfalls der genannten Creative Commons Lizenz, sofern sich aus der Abbildungslegende nichts anderes ergibt. Sofern das betreffende Material nicht unter der genannten Creative gesetzlichen Vorschriften erlaubt ist, ist für die oben aufgeführten Weiterverwendungen des Materials die Einwilligung des jeweiligen Rechteinhabers einzuholen. Weitere Details zur Lizenz entnehmen Sie bitte der Lizenzinformation auf http://creativecommons.org/licenses/by/4.0/ deed.de.

Korrespondenzadresse:

Dr. Ricarda Richter-Dennerlein

Institut für Zellbiochemie

Universitätsmedizin Göttingen

Humboldtallee 23

D-37073 Göttingen

ricarda.richter@med.uni-goettingen.de

http://biochemie.uni-goettingen.de/index. php?id=865

Elisa Hanitsch
Jahrgang 1990. 2011-2014 Biotechnologiestudium (B. Eng.) an der Ernst-Ab-
be-Hochschule Jena. 2014-2016 Studium der Molecular Medicine (M. Sc.) an
der Universität Göttingen. Seit 2016 Doktorandin an der Universitätsmedizin
Göttingen.

